\section{Newborns reach out}

It is widely accepted that neurogenesis takes place in the adult hippocampal dentate gyrus, but questions remain about the functional role of newborn neurons. Previous studies have shown that newborn hippocampal cells receive connections from cortical axons and local interneurons; now, Gage and colleagues demonstrate that the axons of adult-born neurons in turn also form

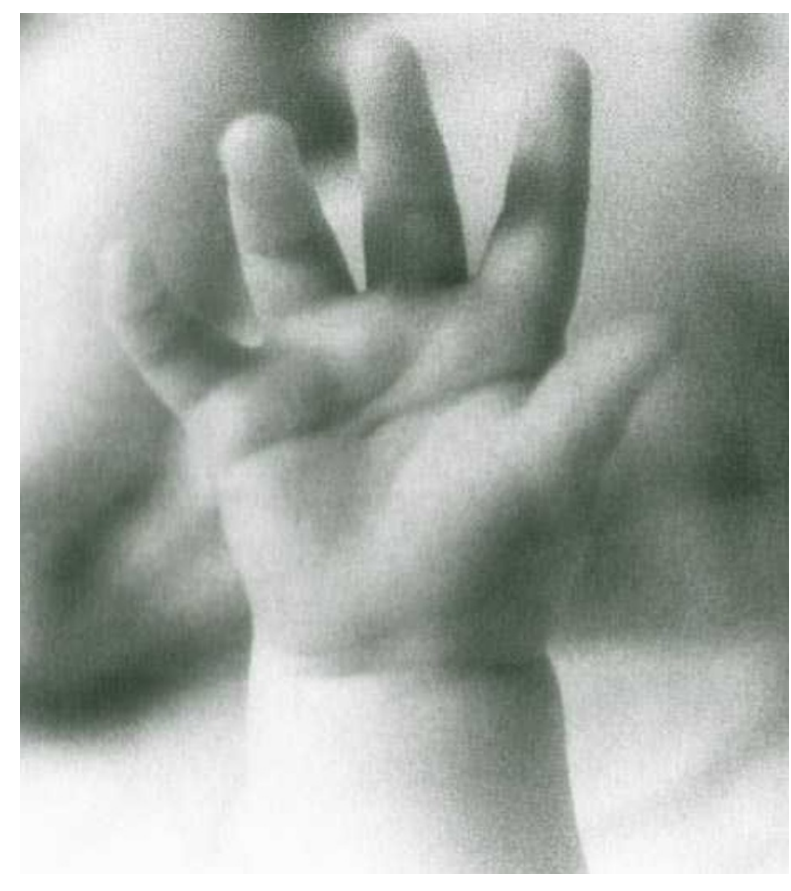

functional 'output' synapses with other hippocampal neurons.

The authors first sought morphological evidence that newborn cells have output synapses. After labelling newborn cells by retroviral delivery of a fluorescent reporter gene, they used immuno-electron microscopy and serial sectioning to study the axonal boutons of the labelled cells and found that they formed synapses with neurons in the hippocampal CA3 and hilus. Interestingly, the connections with CA3 neurons changed over time: the newborn neurons initially formed synapses on to dendritic shafts but later mainly synapsed with dendritic spines and thorny excrescences.

The authors next investigated whether the synapses formed by the newborn neurons were functional. They again used a retrovirus, but this time to deliver not only a fluorescent reporter gene but also the gene that encodes channelrhodopsin 2 (chr2); this approach allowed them to activate the newborn cells in hippocampal slices with blue light. They then used electrophysiology to measure the effect of activation of the newborn cells on their target neurons (including interneurons in the hilus and in the granule cell layer/hilus border, mossy cells in the hilus and pyramidal cells in CA3, as determined by electrophysiological and anatomical measurements). Repetitive blue-light pulses could induce postsynaptic currents in all three types of target cells. Addition of kynurenine, a mixed AMPA ( $\alpha$-amino-3-hydroxy-5-methyl-4isoxazole propionic acid)/NMDA (N-methyl-D-aspartate) receptor antagonist, or of the mGluR agonist DCG-IV, which reduces neurotransmitter release at mossy fibre synapses, blocked evoked postsynaptic currents; this indicates that glutamate is the main neurotransmitter of the newborn hippocampal neurons.

This study provides yet more evidence that adult-born hippocampal neurons are functionally integrated in the hippocampal circuit. Future experiments will be needed to determine the extent to which they contribute to information processing in the hippocampus and how diminished levels of adult neurogenesis during aging affect hippocampal functioning. Leonie Welberg

ORIGINAL RESEARCH PAPER Toni, N. et al. Neurons born in the adult dentate gyrus form functional synapses with target cells. Nature Neurosci. 11, 901-907 (2008) FURTHER READING Gould, E. How widespread is adult neurogenesis in mammals? Nature Rev. Neurosci. 8, 481-488 (2007) 